\title{
DESIGN OF A MICROCONTROLLER BASED SYSTEM TO IMPLEMENT 4-ELECTRODE FOCUSED IMPEDANCE METHOD (FIM)
}

\author{
Aktharuzzaman, Tanvir N Baig and K Siddique-e Rabbani ${ }^{1}$ \\ Department of Physics, University of Dhaka \\ email: shamim17sh@yahoo.com, tnbaig@gmail.com, rabbani@univdhaka.edu
}

\begin{abstract}
Designing of electronic circuitry and development of necessary software has been performed in the present work for a microcontroller based 4-electrode Focused Impedance measurement system. This needs a complex sequential analog switching of constant current ac signal generator and a potential measuring unit to 4 electrodes on the object under study. The performances of the designed system and the developed microcontroller software have been studied using a commercially available simulation software, 'Proteus-7', and the results are very satisfactory.
\end{abstract}

Keywords: Focused Impedance Method, FIM, Electrical Impedance, FIM Instrumentation

\section{INTRODUCTION}

Focused Impedance Method (FIM) for Bio-electric measurements has been conceived and developed in the Biomedical Physics Laboratory of the University of Dhaka (Rabbani et al 1998, 1999, Rabbani and Karal 2008). Three different methods for FIM has been developed, namely, i) 8-electrode FIM, ii) 6electrode FIM and iii) 4-electrode FIM. The basic concept of the 8-electrode FIM involves two tetrapolar measurements taken orthogonally over a central focused zone. The number of required electrodes has been reduced to 6 replacing the 4 potential electrodes by 2 placed at suitable common points of appropriate equipotential lines. In the 4-electrode FIM, four electrodes (shown as 1,2,3,4 in Fig.1) are placed at the corners of a square region which is considered to be the focused zone. Current is first driven through an adjacent electrode pair, say $1 \& 2$, while the potential is measured between the opposite electrode pair, $3 \&$ 4. This impedance measurement has the sensitive zone $\mathrm{E}_{1} \mathrm{~F}_{1} \mathrm{H}_{1} \mathrm{G}_{1}$ shown shaded, between equipotentials passing through the potential measuring electrodes 3 and 4 . Now taking another measurement rotating the electrode configuration by $90^{\circ}$ (current drive: $2 \& 3$, potential: $4 \& 1$ ), the impedance measurement has the sensitive region $\mathrm{E}_{2} \mathrm{~F}_{2} \mathrm{H}_{2} \mathrm{G}_{2}$ shown shaded, between equipotentials passing through the potential measuring electrodes 4 and 1. Summing and averaging the two impedance values thus obtained an enhanced

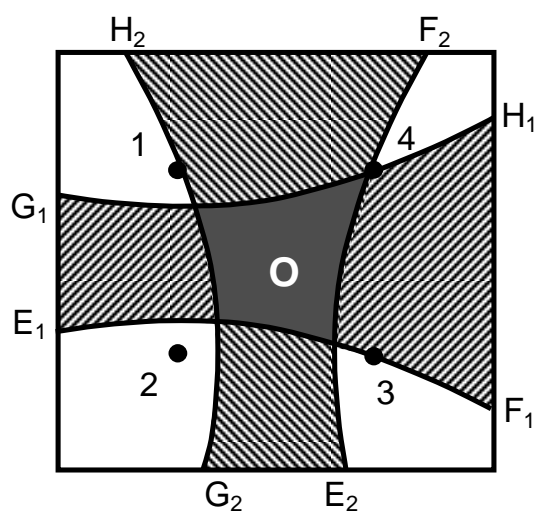

Fig.1: Equipotentials in a 4 electrode FIM showing the focused zone at the centre sensitivity over the central zone ' $\mathrm{O}$ ' (dark shaded) is obtained. The sensitivity of the outer zones will be much less compared to that in the central region of focus. This is the basis of the four-electrode FIM. Another two sets of readings are possible by rotating the current drive and potential measuring electrode combination through further $90^{\circ}$ each and the average of all the four readings will contribute to a reduction of noise and a little improvement in the focusing effect.

The instrumentation for this 4 electrode system will need a constant current source with ac, typically at frequencies between $10 \mathrm{kHz}$ and $100 \mathrm{kHz}$, and a potential measuring circuitry both connected to appropriate pairs of electrodes. From the above description it may be visualized that to implement measurements from all four sides, a complex switching of electrodes to the current source and the

\footnotetext{
${ }^{1}$ Presently at: Department of Biomedical Physics \& Technology, University of Dhaka
} 
potential measuring circuitry will be needed, and all these switches should be able to transfer analogue voltage values. The stages of work involved in developing this instrumentation using analogue switches operated by a microcontroller. The first stage in such a development work is the designing of the logic of the electronic circuitry and of the software for the microcontroller based system, keeping in view several factors such as user friendliness, availability of components, complexity and cost. In the second stage the designed system needs to be simulated using a simulation software package so that the operation can be studied well, whether it can deliver the required performance. In the third stage one goes for real fabrication of the system using hardware electronics and other components. The present work involves the first two stages, that of designing the circuit logic of the 4-electrode FIM system and the development of necessary software. Finally the circuit performance would be studied using a simulation software.

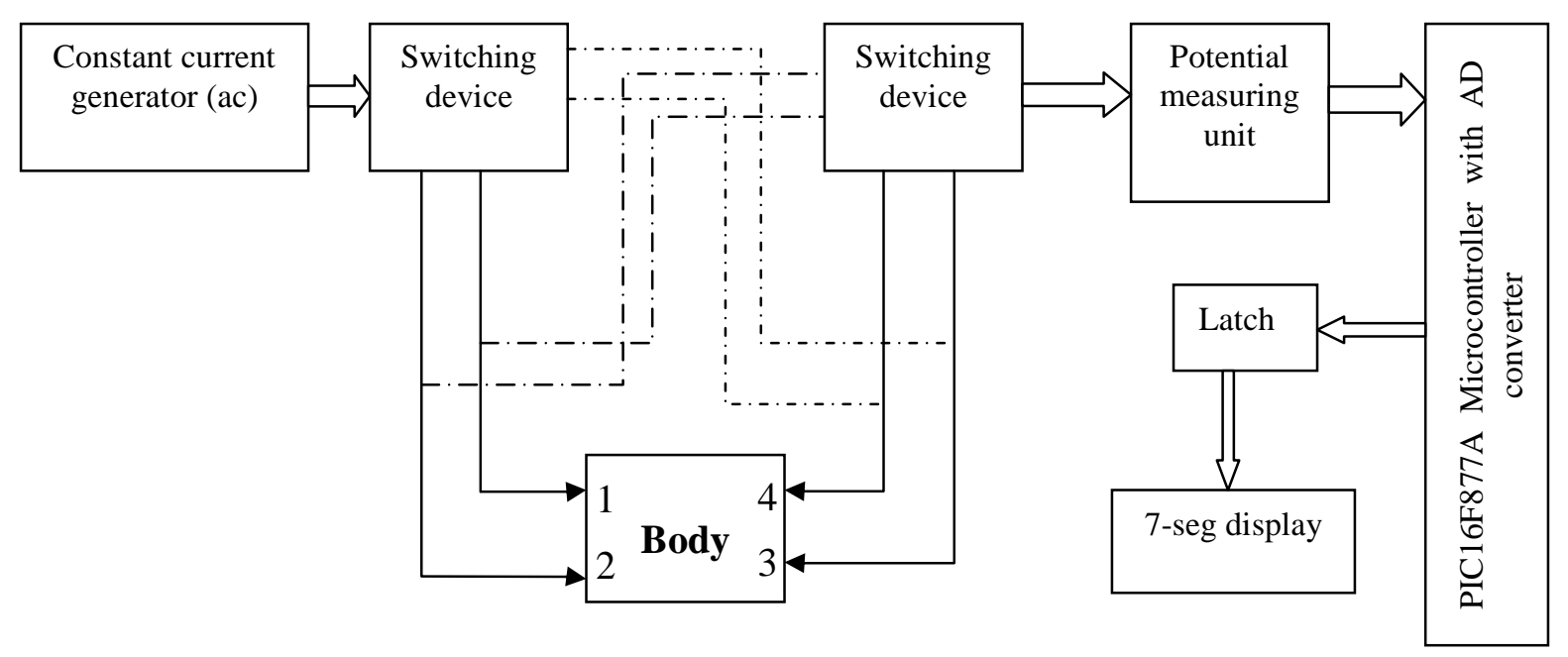

Fig. 2: Block diagram for a microcontroller based 4 electrode FIM system

\section{METHODS}

\subsection{Instrumentation}

A block diagram of the designed system is shown in Fig. 1. Here, four electrodes 1- 4 attached to the body under test are shown at the bottom. A sinusoidal ac constant current generator (with constant amplitude) drives current through two electrodes while the other two electrodes are connected to the inputs of the potential measuring unit. It needs to be mentioned that the potential measurement is done differentially, requiring a common electrode fixed at a suitable location in the body. Since the current is constant, the potential is proportional to the impedance to be measured value ( $Z=V / I$, and with I constant, $Z \propto V$ ). Two analog switch ICs (switching device), under program control of the microcontroller, are employed to switch both the leads of the current drive and the input leads of the potential-measuring unit individually to appropriate electrodes attached to the 'body', indicated by the dotted lines. Thus in one sequence the current generator is connected to electrode pair $1 \& 2$ while electrode pair $3 \& 4$ are taken to the input of the potential measuring circuitry. In the next sequence, the current generator would be connected to electrode pair $2 \& 3$ while pair $4 \& 1$ would be connected to the potential measurement circuitry. Further two sequences will be performed similarly, with current going to electrode pairs $3 \& 4$ and $4 \& 1$ respectively while electrode pairs $1 \& 2$ and $2 \& 3$ respectively will be connected to the potential measuring circuitry. The measured potential is an ac signal which is amplified, filtered, converted to an equivalent dc, and given necessary dc level shift to make the data suitable for the next stage. All this is done by the potential measuring circuitry shown. The analogue dc output is fed to a 
microcontroller having an Analogue to Digital (A/D) converter at the input which converts the signal to the corresponding binary digital value. For the four measurement sets needed for focusing, the appropriate switching is performed by the microcontroller and the corresponding output digital values, which are essentially the impedance values, are stored in its memory. At the end of the sequence, the four stored values are summed, which is proportional to the desired value of focused impedance, and the result is displayed through a 7-segment Display.

Considering availability, cost, and performance, a microcontroller IC with the identification PIC16F877A was chosen for the FIM system. It has a built-in Analogue to Digital (A/D) converter that can be used to advantage. It also has a reduced instruction set, only 35, and as such can be programmed relatively easily. The Analogue switch IC chosen was ADG333A having four SPDT switches. Two of these SPDT switches worked for the current switching while the other two for the potential input switching. Thus a single ADG333A analogue switch IC was adequate for both the switching functions.

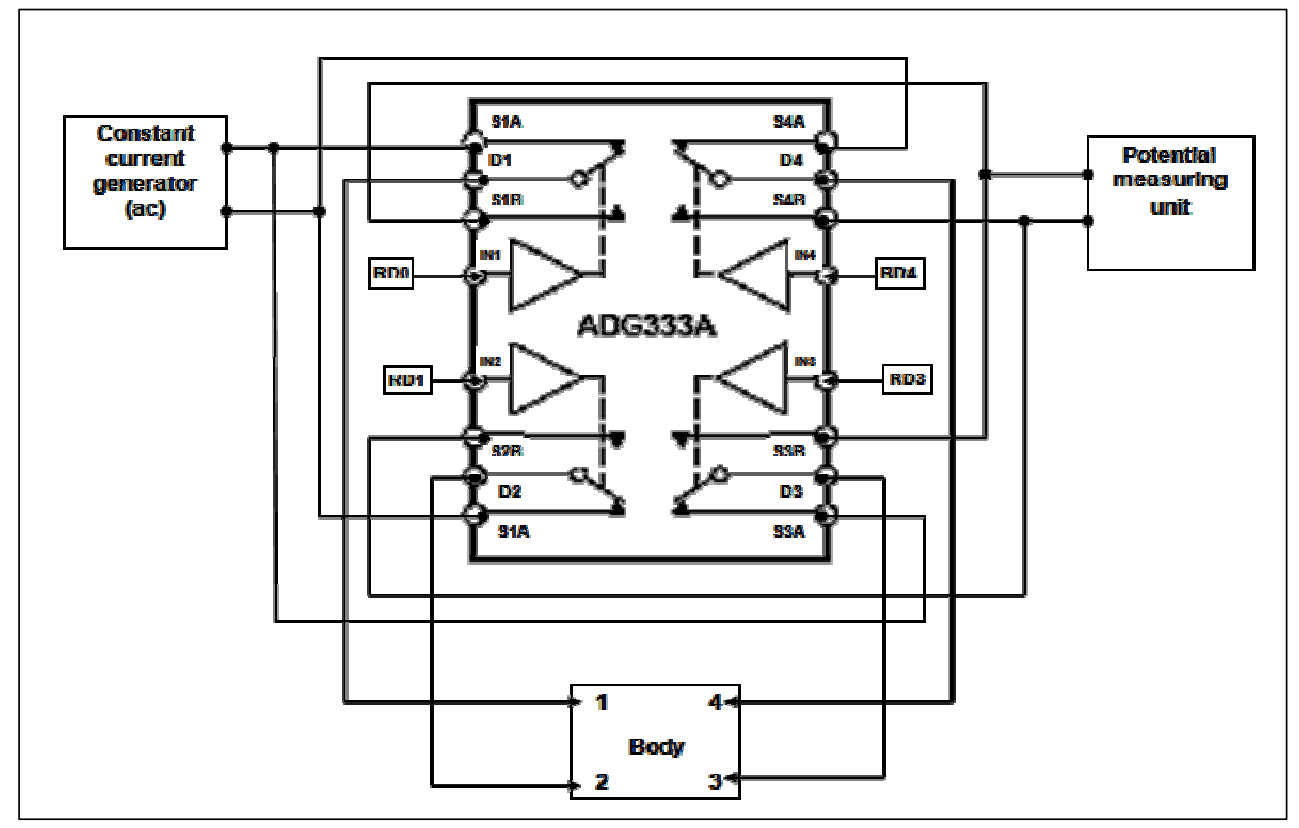

Fig. 3: Details of the switching using ADG333A

Logic signals from the microcontroller select the respective control inputs, RD0 to RD3, of the analogue switch IC to connect the current generator and the potential measurement units to appropriate electrodes on the object (body) under study. After a sequence of four data acquisition steps, corresponding to the four measurement directions at $90^{\circ}$ to each other, all the four data values are summed and the final result is displayed through a 4-digit 7-segment Display in hexadecimal number system. The whole method can be summarized as:

Fig. 3 shows the connections of the ADG333A IC to implement the above functions.

Performing the required complex switching automatically in sequence,

i) Program controlled acquisition of four sequential values of analogue data for each measurement set by first converting these to binary digital data,

ii) Performing the required summing of the four sets of data, and

iii) Displaying the result (summed value) in hexadecimal form through a 4-digit seven segment display.

In order to achieve the sequence of appropriate switching a truth table for the analogue switch was developed and is shown in Table 1. 
Table 1: Truth table for switching of the analogue switch IC

\begin{tabular}{|c|c|c|c|c|c|c|c|c|c|c|c|c|}
\hline sequence & RD0 & RD1 & RD2 & RD3 & S1A & S1B & S2A & S2B & S3A & S3B & S4A & S4B \\
\hline 1 & 1 & 1 & 0 & 0 & C & O & C & O & O & C & O & C \\
\hline 2 & 0 & 1 & 1 & 0 & O & C & C & O & C & O & O & C \\
\hline 3 & 0 & 0 & 1 & 1 & O & C & O & C & C & O & C & O \\
\hline 4 & 1 & 0 & 0 & 1 & C & O & O & C & O & C & C & O \\
\hline Note: & C: Closed & O: open \\
\hline
\end{tabular}

\subsection{Data acquisition and numerical display of result}

The microcontroller PIC16F877A has a built-in 8-channel Analog to Digital Converter. We used only channel 0 for data acquisition. Figure 4 shows the basic circuitry for data acquisition and for displaying of numerical result in hexadecimal using a set of four 7-segment display. This display, available locally, has a built-in binary to hexadecimal converter which makes the system design simple.

The 8-bit PORTB of the microcontroller provides the output binary data. However, the microcontroller provides 10 bit binary data internally while the PORTB can deliver 8 bits only at a time. So we used a multiplexing scheme where we delivered the least significant 8 bits first through PORTB, stored them in an octal latch, and then obtained the higher two bits and stored them in another octal latch. The two latches combined provided a 16 bit output which fed the 16 inputs of the 4-digit hexadecimal 7-segment LED display (with built-in decoder as mentioned before). Of course only the lower 10 bits have been used here. Enable pulses for the operation of the two octal latches in sequence were provided by the microcontroller.

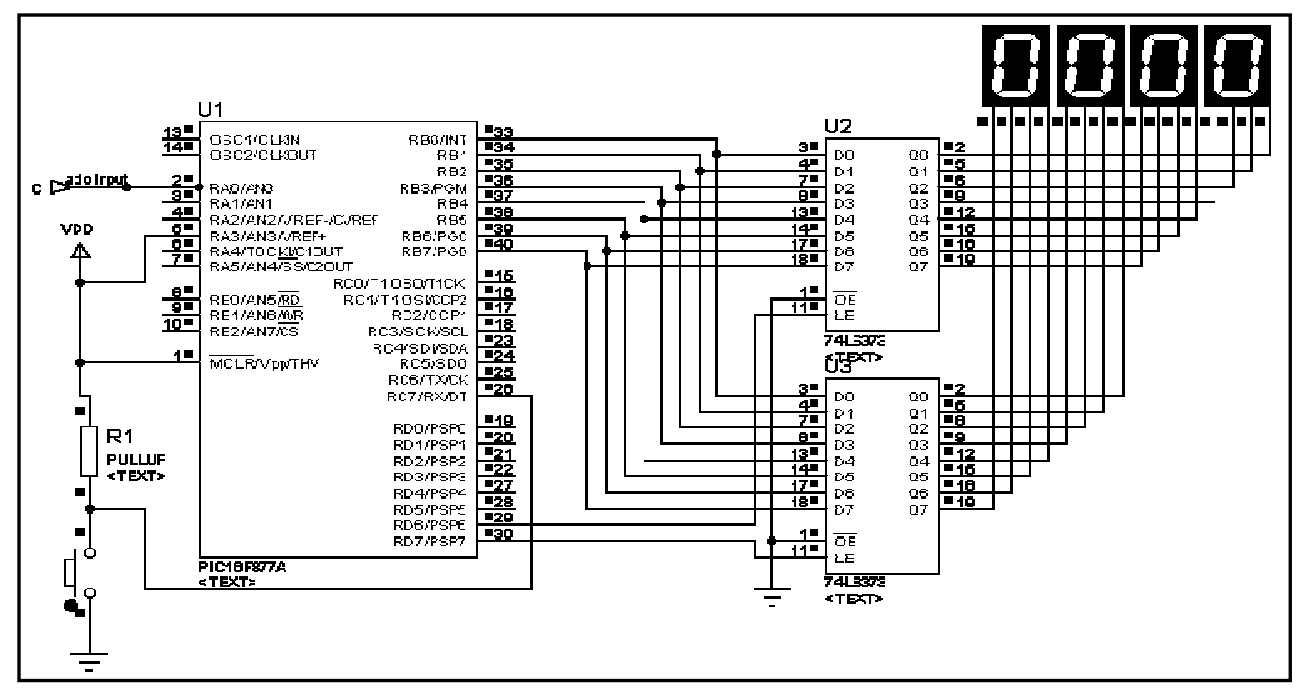

Fig.4: The output latches and display circuitry

\subsection{Software}

A complete program was written in 'Assembly' language for PIC16F877A microcontroller to perform all the above mentioned operations of the FIM properly. 


\section{RESULTS \& OBSERVATIONS}

The performance of the designed system in conjugation with the software developed was tested using a commercially available simulation software package named 'Proteus 7'. A simulated de input was provided from a dc source with a variable output. The sequences of switching, acquisition of four analogue dc data, summation of these values in memory, and displaying the summed value in the display unit were subjected to this test. The result of simulation matched exactly with the desired results.

\section{DISCUSSION}

The previous work on 4 electrode FIM established the method using circuitry previously developed for standard electrical impedance measurements and the sequential switching to electrodes was done manually. This present work is the first effort at an automatic implementation of this technique. The switching, as described before, is rather complex, and would be very difficult using conventional electronic circuitry. Programmed control, either using a PC or a microcontroller, gives a much simpler hardware solution, though the challenge is then transferred to programming. The present work has covered two major steps of the three step programme for development of the full system. The basic design has been made for which choice of devices (microcontroller, analogue switch, etc.) was very important. Data sheets of various models of these devices had to be studied in order to come to a decision. The switching of the current source and the potential measuring unit to electrodes on the object under study was another major challenge which was solved through the development of a truth table. The second step involved development of the appropriate software, which has also been done. The simulation study has demonstrated the success of the circuit design and of the software. So the next step of fabricating the device using hardware components may come up with success without much hitch, it may be hoped. When complete, this microcontroller based 4-electrode FIM would be used for real life physiological study on human subjects and the pave the way to a new dimension of research for the benefit of humanity.

\section{ACKNOWLEDGEMENTS}

The authors wish to thank Mr. Monzurul Alam of Bangladesh Institute of Biomedical Engineering \& Appropriate Technology (Bi-BEAT) for helpful discussions and suggestions.

\section{REFERENCES:}

1. K S Rabbani, M. Sarker, M. H. R. Akond and T. Akter. Focused impedance measurement (FIM) - a new technique with zone localization. Proceedings, X International Conference on Electrical Bioimpedance, Barcelona, Spain: 31-34, 1998,

2. K S Rabbani, M Sarker, M H R Akond and T Akter. Focused Impedance Measurement (FIM) - a new technique with improved zone localization. Annals of the new York Academy of Science, Electrical Bioimpedance Methods: 873, 408-420, 1999.

3. K S Rabbani and M A S Karal, A new four-electrode Focused Impedance Measurement (FIM) system for physiological study, Annals of Biomedical Engg (Springer, USA), Published online. 18 March, 2008, DOI: 10.1007/s10439-008-9470-7, Printed version yet to come out 